University of Nebraska - Lincoln

DigitalCommons@University of Nebraska - Lincoln

Norman R. Simon Papers

Research Papers in Physics and Astronomy

$9-1-1990$

\title{
ON omega CENTAURI AND ITS RRc STARS
}

Norman R. Simon

University of Nebraska - Lincoln, nsimon@unl.edu

Follow this and additional works at: https://digitalcommons.unl.edu/physicssimon

Simon, Norman R., "ON omega CENTAURI AND ITS RRc STARS" (1990). Norman R. Simon Papers. 30.

https://digitalcommons.unl.edu/physicssimon/30

This Article is brought to you for free and open access by the Research Papers in Physics and Astronomy at DigitalCommons@University of Nebraska - Lincoln. It has been accepted for inclusion in Norman R. Simon Papers by an authorized administrator of DigitalCommons@University of Nebraska - Lincoln. 
THE AstrophysiCAL JouRnal, 360:119-128, 1990 September 1

(C) 1990. The American Astronomical Society. All rights reserved. Printed in U.S.A.

\title{
ON $\omega$ CENTAURI AND ITS RRc STARS
}

\author{
NORMAN R. SIMON \\ Department of Physics and Astronomy, University of Nebraska \\ Received 1989 November 13; accepted 1990 March 2
}

\begin{abstract}
Masses, temperatures, and absolute luminosities are proposed for a large sample of RRc stars in the globular cluster $\omega$ Centauri. Our technique uses three observed quantities: period, relative luminosity, and Fourier phase $\phi_{31}$, interpreting them in the light of linear and hydrodynamic pulsation models. The masses we obtain agree with those of the RRd stars in other clusters, and our derived parameters pass a number of tests which are independent of the derivation: (1) we reproduce the observed slope, $\partial \phi_{31} / \partial \log P$, of the $\phi_{31}$-period diagram; (2) the values we obtain for $M, T_{e}$, and $L$ locate the $\omega$ Cen RR $c$ stars in the first overtone instability strip, just where they belong; (3) our derived parameters are shown to be consistent with the fact that $\omega$ Cen contains no RRd stars. However, our results not only disagree with current horizontal branch models, but also imply that the canonical slope of the $\log T_{e}$ versus $B-V$ relation (obtained from static model atmospheres) is too steep by a factor of 2 , at least as applied to the $\mathrm{RR} c$ stars. Some additional results are presented which bear on the confrontation between evolution and pulsation theory: (1) it is shown that the disagreement between the RRd masses and those based on horizontal branch calculations cannot be reconciled by reasonable changes in metallicity or opacity; (2) we find essentially no correlation between the masses of the $\omega$ Cen RRc stars and their metallicities, and we demonstrate that this circumstance cannot be due to late redward evolution by the RR Lyrae stars of lower mass; and (3) the mass-luminosity relation obtained by our method for three RRc field stars is shown to be consistent with the results of a Baade-Wesselink analysis, but not with horizontal branch models. Finally, we briefly discuss the question of globular cluster ages and calculate an age for $\omega$ Centauri based upon its RRc stars.
\end{abstract}

Subject headings: clusters: globular - stars: abundances - stars: pulsation — stars: RR Lyrae

\section{INTRODUCTION}

The RR Lyrae stars in globular clusters have been studied intensively for many years. It is well known that most observed globular clusters containing RR Lyrae stars fall into two distinct groups. In Oosterhoff I (Oo I) clusters, about $75 \%$ of the RR Lyrae stars are fundamental mode pulsators (RR $a b$ stars) with a mean period of about 0.55 , while in Oosterhoff II (Oo II) clusters, the corresponding numbers are $50 \%$ and 0.65 (see, e.g., Rood and Crocker 1989). Furthermore, the Oo II clusters are metal-poor compared with the Oo I clusters. The best illustration of this effect is M15 versus M3. The period dichotomy between the two Oosterhoff groups has proven difficult to understand. Recently, this phenomenon has been formulated more comprehensively as the Sandage period shift effect-namely, that at any temperature, the RR Lyrae periods decrease with increasing metallicity of the cluster (Sandage 1982). The problem with the period shift is that standard evolutionary models cannot reproduce it unless the uncomfortable assumption is made that the helium and metal abundances are anticorrelated.

The rich globular cluster $\omega$ Centauri contains a large number of RR Lyrae stars which encompass a wide range of metallicity and thus overlap the two Oosterhoff groups. For this reason, $\omega$ Cen constitutes a potential testing ground not only for theories of stellar pulsation and evolution, but for globular cluster ages and distances as well. Unfortunately, this cluster also has a number of seemingly contrary properties, including the absence of a luminosity-metallicity correlation and its failure to display either the Oosterhoff dichotomy or the Sandage period-shift effect. These questions are treated in a recent review by Dickens (1989, hereafter D89).
While $\omega$ Cen possesses large samples of both RR $a b$ (fundamental mode) and $\mathrm{RR} c$ (first overtone) pulsators, it seems to lack even a single RR $d$ star (double mode pulsator) (Nemec, Nemec, and Norris 1986). The latter stars have been used in other clusters to determine RR Lyrae masses with the result $M \cong 0.55 M_{\odot}$ in Oo I clusters, $M \cong 0.65 M_{\odot}$ in Oo II clusters (Cox, Hodson and Clancy 1983; Cox 1988). In a recent investigation, Simon (1989, hereafter S89) used hydrodynamic pulsation models and Fourier decomposition to derive a mass range for the $\omega$ Cen $\mathbf{R} R c$ stars that agrees with the RR $d$ masses noted above. The present work will be devoted to an amplification of S89, including its ramifications for the study of $\omega$ Cen in particular, and more generally of the masses, luminosities, and temperatures of RR Lyrae stars.

\section{RESULTS FROM S89}

In S89 a large grid of hydrodynamic $\mathrm{RR} c$ models was compared with a sample of observed stars by means of Fourier decomposition. In this technique (Simon 1988a), the observed and theoretical light curves are fit with Fourier series of the form $A_{0}+\sum_{j} A_{j} \cos \left(j \omega t+\phi_{j}\right)$, and the comparison made in terms of the quantities $R_{j 1}=A_{j} / A_{1}$ and $\phi_{j 1}=\phi_{j}-j \phi_{1}$. Here as in S89, we focus on the Fourier phase parameter $\phi_{31}=$ $\phi_{3}-3 \phi_{1}$. A discussion of other parameters as well as details of the hydrodynamic models themselves have been given elsewhere (Simon 1990).

A least-squares fit made to the hydrodynamic models in S89 yielded the result:

$$
\log L=0.105 \phi_{31}+1.814 \log M-0.081 \log Y+1.692 .
$$

The relatively small standard deviation of this fit $(0.03$ in $\log L)$ 


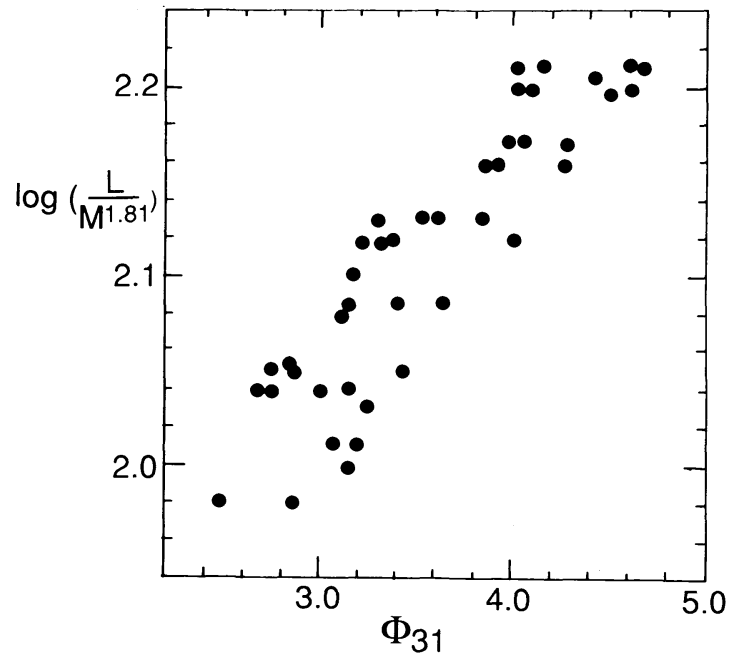

FIG. 1.-The quantity $\log L-1.81 \log M$ vs. $\phi_{31}$ (see eq. [1]) for the hydrodynamic models.

and the weak helium dependence indicate that $\phi_{31}$ is a measure of $L / M^{1.81}$. This relationship is illustrated in Figure 1.

A second fit to the models, but now introducing the pulsation period as a new variable, gave the expression:

$$
\phi_{31}=6.013 \log P-7.398 \log M+5.098 \log Y+7.571 \text {. }
$$

As explained in S89, the strong helium dependence in equation (2) is due to the fact that $Y$ influences the theoretical blue edges and thus the model temperatures, which in turn strongly affect the periods.

The direct observational parameters relating to equations (1) and (2) are the Fourier phase $\phi_{31}$ and the period $P$. In addition, for a given cluster, one can obtain the luminosity of each star to within a common constant. For the case of $\omega$ Centauri, these observational quantities have been given by Petersen (1984) based upon the photographic RR Lyrae data of Martin (1938). Applying the Fourier decomposition technique to Martin's light curves, Petersen found clear evidence for a gradual rise of $\phi_{31}$ with increasing period among the $\mathrm{RR} c$ stars.

While the $\phi_{31}$ progression was tentatively attributed by Petersen (1984) to a period resonance analogous to that appearing among the classical Cepheids (Simon and Schmidt 1976), it can be seen from equation (2) that such an assumption is not necessary. The increase of $\phi_{31}$ with period occurs in standard, nonresonant $\mathrm{RR} c$ models. In fact, $\mathrm{S} 89$ was able to show that the coefficient of $\log P$ in equation (2) agrees very well with that emerging from an analysis of the RRc stars in $\omega$ Cen. This argument is summarized briefly in what follows.

Applying equation (1) to observed values of $\phi_{31}$ and relative luminosity for the RR $c$ stars in $\omega$ Cen, we find the mass of each star to within an arbitrary constant, i.e.,

$$
\log M=\log M_{f}+\Delta \log M,
$$

where $\Delta \log M$ is obtained from equation (1) and $\log M_{f}$ is a constant related to $\omega$ Centauri's distance. Using these masses, we then make a least-squares fit to the $\omega$ Cen RR $c$ stars in terms of three variables: $\phi_{31}, \log P$, and $\log M$. The coefficient of $\log P$ emerging from this fit has the value 5.83, quite close to the corresponding quantity in equation (2).

Furthermore, it was shown by $\mathbf{S} 89$ that although the coefficient of $\log M$ in equation (2) diverged somewhat from its observational counterpart, it could be brought into line pro- vided one assumed a relationship of the following form between the mass of an RRc star and its helium abundance:

$$
\log Y=-0.63 \log M+h_{2} \text {, }
$$

where $\log Y$ refers to the pulsating layers of the RR Lyrae envelope and not necessarily to the main-sequence progenitor. The value of the constant $h_{2}$ is fixed once a choice is made for the constant $\log M_{f}$ in equation (3), or vice versa. In addition, since this choice fixes the mass, luminosity, and helium abundance of each $\mathrm{RRc}$ star in the cluster, it also specifies the average value of each of these parameters as well as a distance modulus for $\omega$ Cen as a whole. Thus, choosing a value for log $M_{f}\left(\right.$ or $\left.h_{2}\right)$ is equivalent to specifying $\omega$ Cen's distance.

Finally, it was demonstrated by $\mathrm{S} 89$ that the choice for $h_{2}$ which yields reasonable helium abundances $(0.24 \leq Y \leq 0.29)$ also gives a mass range $0.49 \leq M / M_{\odot} \leq 0.67$, approximately in line with the RRd masses obtained from other clusters.

\section{MASS AND TEMPERATURE}

We now obtain temperatures for the $\omega$ Cen RR $c$ stars using the following fit to the linear, nonadiabatic counterparts of our hydrodynamic models:

$\log P=0.8018 \log L-0.6043 \log M$

$$
-3.3455 \log T_{e}+10.933,
$$

where $P$ is the (overtone) period and $L, M$, and $T_{e}$ are model parameters. This equation is merely an expression of the period/mean density law. Since values of $P, L$, and $M$ are known or derived for each $\omega$ Cen star, the temperature is easily calculated from equation (5).

Table 1 shows results from four choices of the arbitrary parameter $\log M_{f}$ in equation (3) or, equivalently, $h_{2}$ in equation (4). The rows give, in order, $\log M_{f}$ and $h_{2}$, and then the means and ranges of mass, helium abundance, luminosity, and temperature, and finally, the mean distance modulus $\langle m-M\rangle$ (S89). The preferred choice mentioned in the previous section corresponds to case 2 . In Table 2 we list parameters according to case 2 for a "reduced" sample (see S89) of $47 \mathrm{RR} c$ stars in $\omega$ Cen. The period, relative luminosity, and Fourier phase $\phi_{31}$ are observed quantities, while the mass, temperature, and helium abundance have been determined as described above. The stars are identified by their standard numbers (see, e.g., Petersen 1984).

In Figure 2 we plot $\phi_{31}$ versus period for the sample in Table 2. The mass (times 100) is indicated for each star. Had we chosen a different case from Table 1 , this would change the

TABLE 1

Parameters For $\omega$ Centauri RR $c$ Stars

\begin{tabular}{ccccc}
\hline \hline & \multicolumn{5}{c}{ CASE } \\
\cline { 2 - 5 } PARAMETER & 1 & 2 & 3 & 4 \\
\hline $\log M_{f} \ldots \ldots \ldots$ & -0.260 & -0.187 & -0.125 & -0.071 \\
$h_{2} \ldots \ldots \ldots \ldots \ldots$ & -0.884 & -0.732 & -0.603 & -0.490 \\
$\langle M\rangle \ldots \ldots \ldots \ldots$ & 0.48 & 0.57 & 0.66 & 0.75 \\
$M($ range) $\ldots \ldots$. & $0.42-0.57$ & $0.49-0.67$ & $0.57-0.78$ & $0.65-0.88$ \\
$\langle Y\rangle \ldots \ldots \ldots \ldots$ & 0.21 & 0.26 & 0.32 & 0.39 \\
$Y($ range) $\ldots \ldots$. & $0.19-0.23$ & $0.24-0.29$ & $0.29-0.35$ & $0.35-0.43$ \\
$\langle\log L\rangle \ldots \ldots \ldots$ & 1.58 & 1.71 & 1.81 & 1.90 \\
$\log L$ (range) $\ldots$. & $1.48-1.68$ & $1.60-1.81$ & $1.71-1.91$ & $1.80-2.00$ \\
$\left\langle T_{e}\right\rangle \ldots \ldots \ldots \ldots$ & 6770 & 7030 & 7260 & 7468 \\
$T_{e}($ range) $\ldots \ldots$. & $6490-7090$ & $6750-7370$ & $6970-7610$ & $7170-7830$ \\
$\langle m-M\rangle \ldots \ldots$ & 13.9 & 14.2 & 14.4 & 14.7 \\
\hline$m$
\end{tabular}


TABLE 2

PARAMETERS For INDIVIDUAL $\omega$ Centauri RR $c$ Stars ACCORDING TO CASE 2

\begin{tabular}{|c|c|c|c|c|c|c|}
\hline $\begin{array}{c}\text { Star } \\
\text { Number }\end{array}$ & $\begin{array}{c}P \\
\text { (days) }\end{array}$ & $\phi_{31}$ & $\log \left(L / L_{\odot}\right)$ & $M / M_{\odot}$ & $T_{e}$ & $Y$ \\
\hline 98. & 0.281 & 2.80 & 1.637 & 0.604 & 7327 & 0.254 \\
\hline $19 .$. & 0.300 & 3.37 & 1.600 & 0.535 & 7192 & 0.274 \\
\hline $121 \ldots$ & 0.304 & 2.71 & 1.715 & 0.673 & 7322 & 0.238 \\
\hline $127 \ldots$ & 0.305 & 3.44 & 1.686 & 0.590 & 7369 & 0.258 \\
\hline 16. & 0.330 & 3.13 & 1.702 & 0.627 & 7185 & 0.248 \\
\hline $124 \ldots$ & 0.332 & 3.03 & 1.698 & 0.632 & 7148 & 0.247 \\
\hline $137 \ldots$ & 0.334 & 2.95 & 1.719 & 0.665 & 7168 & 0.242 \\
\hline$\ldots \ldots$ & 0.335 & 4.14 & 1.640 & 0.509 & 7175 & 0.283 \\
\hline 82. & 0.336 & 3.30 & 1.694 & 0.607 & 7157 & 0.253 \\
\hline$\ldots \ldots$ & 0.338 & 3.37 & 1.735 & 0.633 & 7254 & 0.247 \\
\hline ....... & 0.341 & 3.73 & 1.685 & 0.568 & 7178 & 0.264 \\
\hline $126 .$. & 0.342 & 3.44 & 1.694 & 0.596 & 7143 & 0.256 \\
\hline$\ldots \ldots$ & 0.345 & 3.15 & 1.682 & 0.610 & 7050 & 0.253 \\
\hline ....... & 0.357 & 3.97 & 1.697 & 0.559 & 7150 & 0.267 \\
\hline$\ldots \ldots$ & 0.358 & 2.93 & 1.711 & 0.650 & 7003 & 0.243 \\
\hline $158 \ldots$ & 0.367 & 4.01 & 1.755 & 0.596 & 7229 & 0.256 \\
\hline$\ldots \ldots$ & 0.370 & 2.90 & 1.731 & 0.669 & 6974 & 0.238 \\
\hline ........ & 0.373 & 3.60 & 1.727 & 0.607 & 7060 & 0.253 \\
\hline$\ldots \ldots$ & 0.375 & 3.56 & 1.690 & 0.584 & 6959 & 0.260 \\
\hline$\ldots \ldots$ & 0.375 & 3.98 & 1.718 & 0.572 & 7093 & 0.263 \\
\hline $14 \ldots \ldots \ldots \ldots$ & 0.377 & 4.21 & 1.693 & 0.538 & 7061 & 0.273 \\
\hline $36 \ldots \ldots \ldots \ldots$ & 0.380 & 3.91 & 1.714 & 0.574 & 7045 & 0.262 \\
\hline $72 \ldots$ & 0.385 & 3.91 & 1.706 & 0.569 & 7000 & 0.264 \\
\hline$\ldots \ldots \ldots$ & 0.386 & 4.29 & 1.652 & 0.507 & 6930 & 0.284 \\
\hline $153 \ldots \ldots \ldots \ldots$ & 0.387 & 3.58 & 1.702 & 0.591 & 6927 & 0.258 \\
\hline ........ & 0.387 & 3.40 & 1.702 & 0.605 & 6897 & 0.254 \\
\hline ....... & 0.387 & 3.91 & 1.706 & 0.569 & 6988 & 0.264 \\
\hline $81 \ldots \ldots \ldots \ldots$ & 0.389 & 4.26 & 1.685 & 0.530 & 6983 & 0.276 \\
\hline $70 \ldots \ldots \ldots \ldots$ & 0.391 & 4.06 & 1.701 & 0.555 & 6982 & 0.268 \\
\hline $136 \ldots \ldots \ldots \ldots$ & 0.392 & 4.00 & 1.804 & 0.635 & 7204 & 0.246 \\
\hline ........ & 0.392 & 3.69 & 1.726 & 0.600 & 6971 & 0.255 \\
\hline $39 \ldots \ldots \ldots \ldots$ & 0.393 & 4.44 & 1.689 & 0.520 & 7000 & 0.279 \\
\hline $87 \ldots \ldots \ldots \ldots$ & 0.397 & 3.72 & 1.710 & 0.586 & 6915 & 0.259 \\
\hline$\ldots \ldots \ldots$ & 0.396 & 4.38 & 1.693 & 0.527 & 6986 & 0.277 \\
\hline $160 \ldots$ & 0.397 & 4.63 & 1.697 & 0.512 & 7029 & 0.282 \\
\hline $30 \ldots \ldots \ldots \ldots$ & 0.405 & 3.99 & 1.726 & 0.577 & 6954 & 0.261 \\
\hline $95 \ldots \ldots \ldots \ldots$ & 0.405 & 4.66 & 1.680 & 0.500 & 6957 & 0.286 \\
\hline ........ & 0.407 & 4.18 & 1.693 & 0.541 & 6895 & 0.272 \\
\hline $155 \ldots \ldots \ldots \ldots$ & 0.414 & 3.85 & 1.710 & 0.576 & 6847 & 0.262 \\
\hline$\ldots \ldots \ldots$ & 0.422 & 4.71 & 1.713 & 0.517 & 6955 & 0.280 \\
\hline $75 \ldots \ldots \ldots \ldots$ & 0.422 & 4.12 & 1.718 & 0.562 & 6867 & 0.266 \\
\hline $77 \ldots$ & 0.426 & 4.94 & 1.700 & 0.494 & 6943 & 0.288 \\
\hline $147 \ldots \ldots \ldots \ldots$ & 0.423 & 3.36 & 1.751 & 0.646 & 6819 & 0.244 \\
\hline $24 \ldots \ldots \ldots \ldots$ & 0.462 & 4.57 & 1.713 & 0.527 & 6745 & 0.277 \\
\hline $123 \ldots$ & 0.474 & 4.95 & 1.709 & 0.499 & 6745 & 0.287 \\
\hline $47 .$. & 0.485 & 5.13 & 1.770 & 0.525 & 6863 & 0.277 \\
\hline $68 \ldots \ldots \ldots \ldots$ & 0.535 & 5.42 & 1.806 & 0.529 & 6794 & 0.276 \\
\hline
\end{tabular}

mass scale but leave the relative masses intact. Figure 2 clearly shows the tendency of the higher mass stars to sink in the $\phi_{31}$-period diagram and that of the lower mass stars to rise. Furthermore, the stars with the largest values of $\phi_{31}$ all have low masses.

Turning now to the temperatures, we see that case 2, which has already yielded agreement with the RR $d$ masses for plausible helium abundances, also gives a very reasonable temperature range for the $\mathbf{R R} c$ stars. Let us pursue this subject in more detail. Color temperatures for the $\omega$ Cen RR Lyrae stars have been given by Butler, Dickens and Epps (1978, hereafter BDE). These temperatures were obtained from observed $B-V$ colors using an extensive grid of model atmosphere and synthetic spectrum calculations. Table 1 of BDE contains 29 stars in common with our reduced $\omega$ Cen sample. ${ }^{1}$ Although a

${ }^{1}$ This includes star no. 24, which is given as R Rab in BDE but seems clearly to be an $\operatorname{RR} c$ star according to the analysis of Peterson (1984).

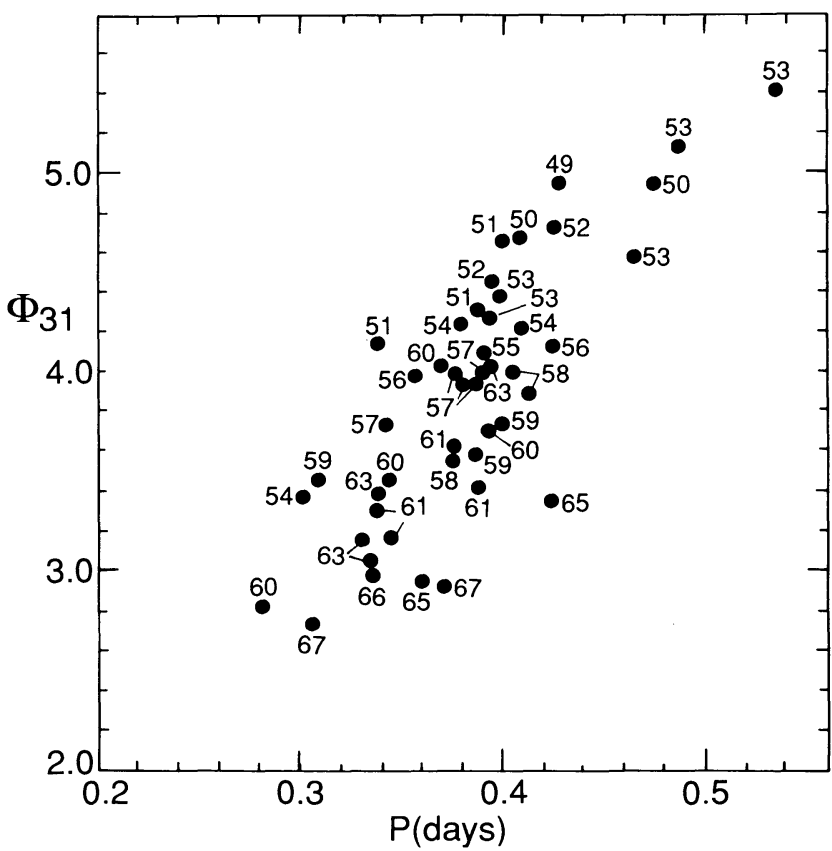

FIG. 2.- $\phi_{31}$ vs. period for $47 \omega$ Cen RR $c$ stars. The number beside each point indicates the mass (times 100 ) according to case 2 (see Table 2).

number of these stars lack observed $[\mathrm{Fe} / \mathrm{H}]$ values and thus have temperatures derived using a mean $\langle[\mathrm{Fe} / \mathrm{H}]\rangle=-1.43$, this should not constitute too serious a problem, since the metallicity dependence is fairly weak at the higher temperature range occupied by the $\mathrm{RR} c$ stars (see BDE).

Figure 3 shows a plot of $\Delta \log T_{e}$ (with the sense, case 2 minus BDE) versus $\log P$ for the 29 common stars. One notes that the BDE temperatures are higher than their case 2 counterparts in a large majority of instances, and that there is a trend with period, such that $\Delta \log T_{e}$ narrows and even reverses as the period increases. The bulk of the difference between the case 2 and BDE temperatures, however, occurs for the hotter stars. This is illustrated in Figure 4, which displays magnitude-temperature diagrams for the reduced $\omega$ Cen sample (S89) according to case 2, and for the $\mathrm{RR} c$ stars that have temperatures published by BDE. Although the ordinates are somewhat different, Figure 4 clearly shows the extension of the BDE temperatures blueward of the domain occupied in the case 2 version. The result is a compression in temperature of the case $2 \mathrm{RR} c$ instability strip as compared with BDE.

One possible test of the case 2 temperatures involves their relationship with the colors of the $\omega$ Cen $\mathrm{RR} c$ stars. We recall that our temperatures are determined from three observed quantities - period, relative luminosity, and $\phi_{31}$-interpreted by means of the hydrodynamic models. This determination is completely independent of the colors. If our pulsation theory were far off the mark, one might expect an arbitrary, perhaps even ludicrous, relation between the temperatures derived from that theory and the observed colors. In Figure 5, we make this test in the form of a plot of the case 2 temperatures versus the values of $B-V$ given by $\mathrm{BDE}$ for the common 29 star sample. We note that despite the scatter, Figure 5 has a reasonable look, with $\log T_{e}$ clearly decreasing as $B-V$ grows. However, a fit to the 29 points yields $\log T_{e}=-0.16(B-V)+3.88$, whereas the $\mathrm{BDE}$ temperatures give $\log T_{e}=-0.33$ $(B-V)+3.93$. Thus the pulsational (case 2) temperatures imply a slope $d \log T_{e} / d(B-V)$, which is in absolute value less 


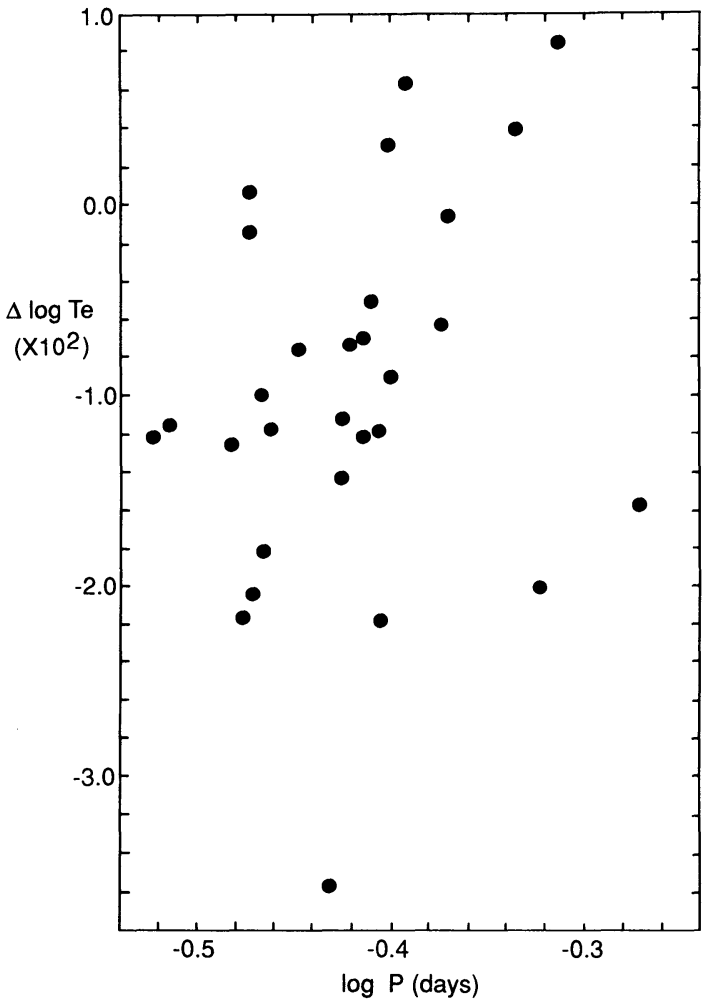

FIG. 3.-The quantity $\Delta \log T_{e}=\log T_{e}$ (case 2) $-\log T_{e}$ (BDE) vs. period for the 29 star common $\mathrm{RR} c$ sample.

than half as large as that determined from static model atmospheres. (A crude estimate from the tables of VandenBerg and Bell 1985 also yields a "large" value for this slope, namely $\left|d \log T_{e} / d(B-V)\right| \gtrsim 0.4$.

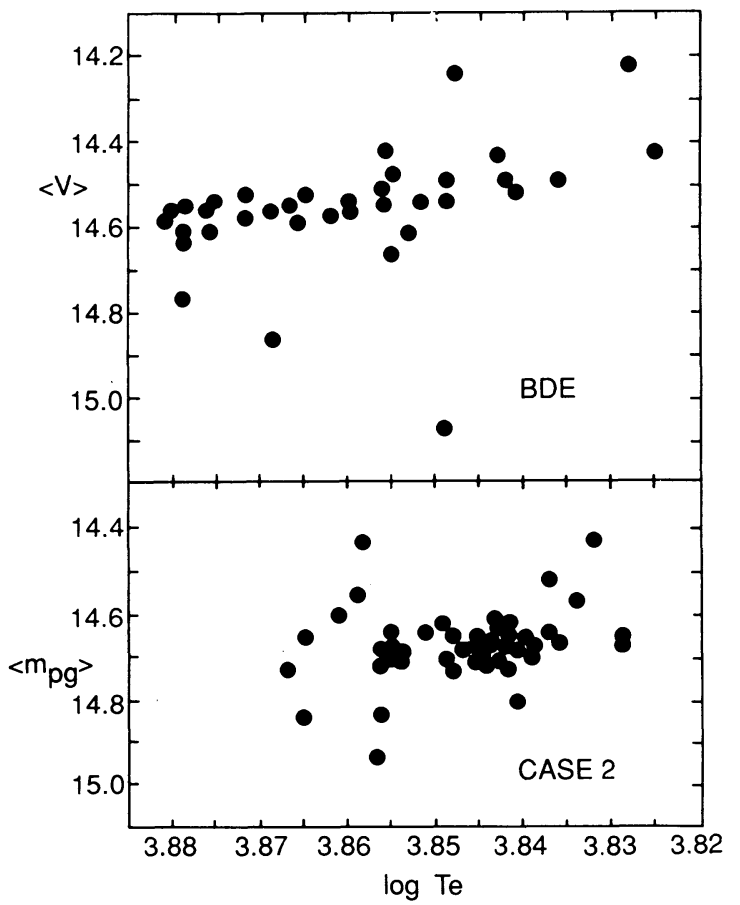

Fig. 4.-A plot for the RR $c$ stars of $\langle V\rangle$ vs. $\log T_{e}$ according to BDE, and $\left\langle m_{\mathrm{pg}}\right\rangle$ vs. $\log T_{e}$ according to case 2 .

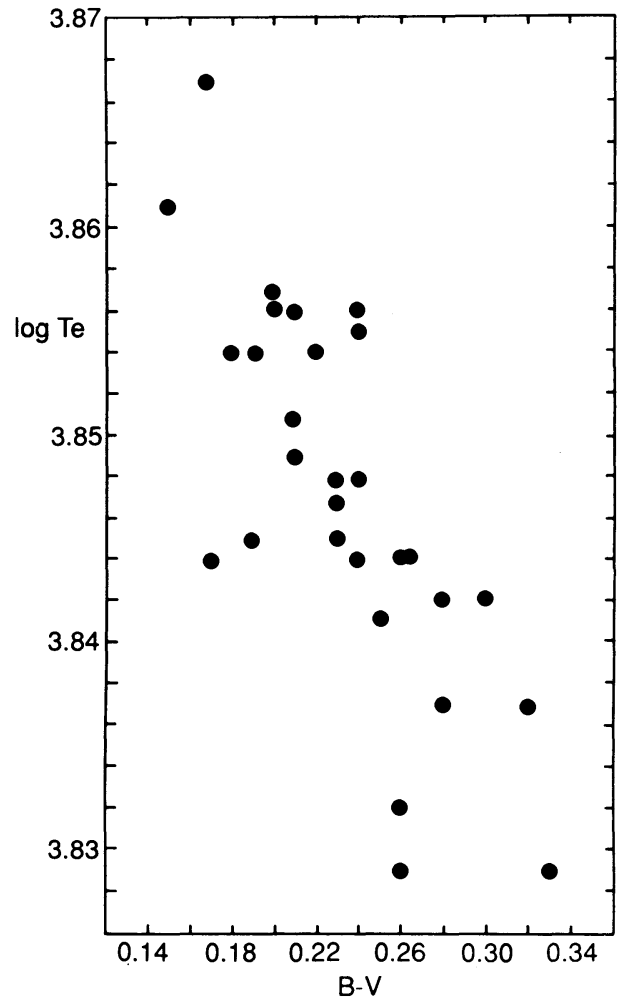

Fig. 5.- $\log T_{e}$ (case 2) vs. $B-V$ (observed) for $\omega$ Cen RR $c$ stars

Let us now recall that we can find in Table 2 masses, luminosities, and temperatures according to case 2 for the reduced $\omega$ Cen sample of 47 RRc stars. For the 29 stars in common with BDE, we have BDE temperatures and can determine "BDE masses and luminosities" as follows. Choose a distance to $\omega$ Centauri. This changes the relative luminosities to absolute luminosities, upon which the masses may be determined directly from equation (5). For further comparison of our results with BDE, the obvious choice of an $\omega$ Cen luminosity scale (i.e., distance) would seem to be that of case 2, which yields for the $47 \mathrm{RR} c$ stars an average luminosity $\left\langle\log L / L_{\odot}\right\rangle=1.71$ (see Table 1). However, in that case the average "BDE mass" for the 29 star common sample is found to be $\left\langle M / M_{\odot}\right\rangle_{\mathrm{BDE}}=0.49$, a value which seems much too small. For this reason, we shall use for the calculation of BDE masses a larger scale which corresponds to a mean luminosity $\left\langle\log L / L_{\odot}\right\rangle=1.76$ for the $\omega$ Cen stars. The latter number is very close to the one adopted by D89 based upon a mainsequence distance calibration. With this choice, one obtains $\left\langle M / M_{\odot}\right\rangle_{\mathrm{BDE}}=0.59$, a value which (perhaps fortuitously) agrees rather well with the mean mass emerging from case 2 (which nonetheless has a smaller mean luminosity).

With masses, luminosities, and temperatures thus in hand for 47 stars according to case 2, and 29 stars according to BDE (for the latter using $\left\langle\log L / L_{\odot}\right\rangle=1.76$ ), we can construct for each object a linear nonadiabatic pulsation model. For the case 2 sample, the helium abundance for each star is that given in Table 2, while for the BDE sample, we use $Y=0.30$. In all cases, the metal abundance is taken to be $Z=0.001$. Figure 6 shows a plot of the linear first overtone driving $\eta_{1}$ (in percent per period, times $10^{3}$ ) versus period. The dots are the case 2 points, and the open circles represent BDE. It is seen that 11 of the $29 \mathrm{BDE}$ stars are linearly stable, some of them exceedingly 


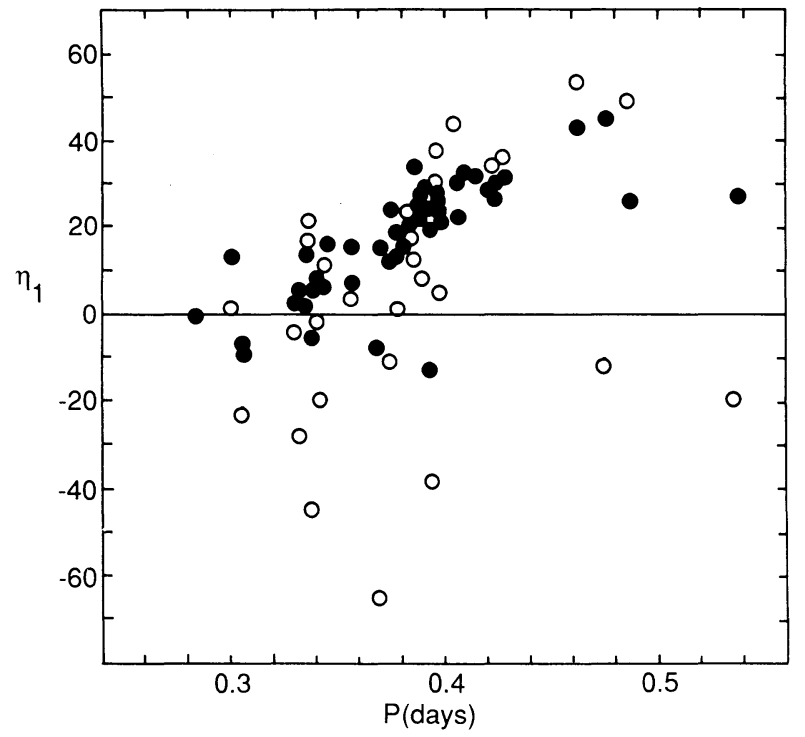

FIG. 6.-First overtone driving $\eta_{1}$ vs. period for RR $c$ models with parameters according to case 2 (dots) and BDE (open circles). The domain below the solid line corresponds to models which are pulsationally stable.

so. This is in contrast with the case 2 parameters, according to which only six of 47 objects are beyond the overtone blue edge and none by a large margin. Once more, we see the consistency of the picture provided by pulsation theory: the parameters we derive for the $\omega$ Cen $\mathrm{RR} c$ stars put these objects in the overtone instability region, just where they ought to be.

What if we had chosen the case 2 luminosity scale $\left(\left\langle\log L / L_{\odot}\right\rangle=1.71\right)$ for the BDE calculations? The answer turns out to be that the BDE stars would have been even more stable! This would also have been the case had we employed a helium abundance less than $Y=0.30$. Thus, the BDE temperature scale leads in too many cases to a contradiction: stars which are pulsating in the first overtone have derived parameters which place them in the (nonpulsating) region beyond the overtone blue edge.

At this point, it should be noted that the BDE study is over a decade old. The results of very recent work on $\omega$ Centauri have been described by $\mathrm{D} 89$. In this investigation, new $B$ and $V$ data were obtained for the $\omega$ Cen RR Lyrae stars, and new temperatures were calculated using the models of VandenBerg and Bell (1985). According to D89, these temperatures are about 150-200 K cooler than their BDE equivalents, a circumstance which suggests the possibility of better agreement with the present case 2. However, a closer examination seems to destroy this hope.

In Figure 7 we present a period-temperature plot for the present case 2 sample (dots), and for $16 \omega$ Cen RRc stars, transcribed from Figure 18 of D89 (open circles). The latter stars seem to describe in Figure 7 a much shallower slope, with the sense that $T(\mathrm{D} 89)>T$ (case 2) at short periods, and $T(D 89) \lesssim$ (case 2 ) at long periods. This is qualitatively the same trend displayed by $T(\mathrm{BDE})$ versus $T$ (case 2 ), as depicted in Figure 3. Thus it appears likely that the cooler temperatures found by D89 must on balance refer to the RRab (rather than to the $\mathrm{RR} c$ ) sample.

We recall that adoption of a mean luminosity $\left\langle\log L / L_{\odot}\right\rangle=$ 1.76 led via equation (5) to a mean mass of $0.59 M_{\odot}$ for the BDE RRc stars. On the other hand, D89 has employed a period/mean density relation similar to equation (5) (Lub 1987)

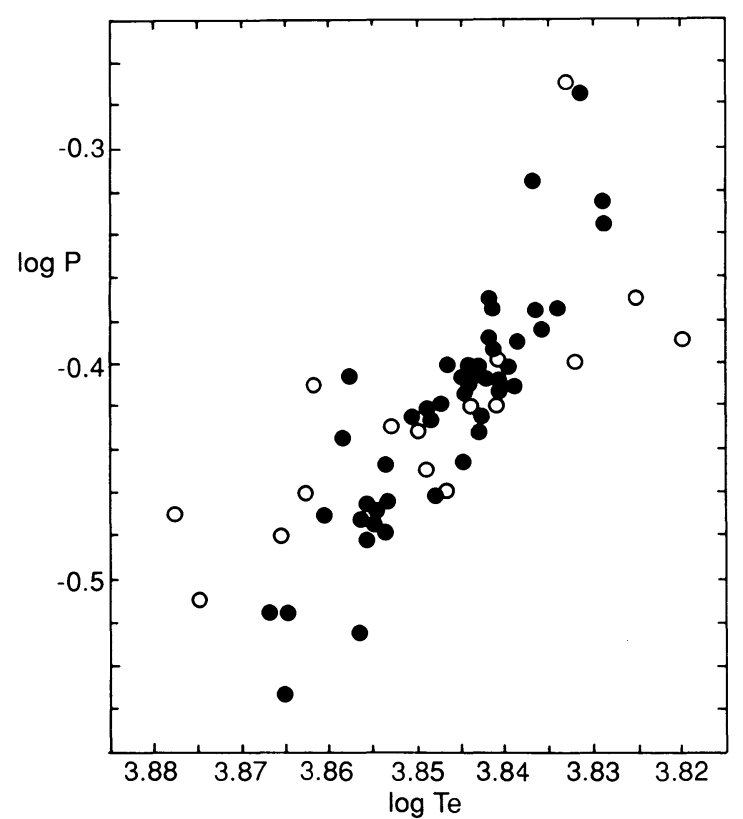

Fig. 7. - Period vs. temperature for $\omega$ Cen RR $c$ stars according to case 2 (dots) and D89 (open circles).

to obtain for $\left\langle\log L / L_{\odot}\right\rangle=1.77$, a mean RR Lyrae mass of $0.73 M_{\odot}$ ! If one uses the case 2 luminosity scale $(<\log$ $\left.\left.L / L_{\odot}\right\rangle=1.71\right)$ in the D89 calculation, the result is $\left\langle M / M_{\odot}\right\rangle=0.62$, as compared with the case 2 mean mass of $0.57 M_{\odot}$. The mean RR Lyrae masses obtained in the various treatments are summarized for convenience in Table 3.

Because the D89 review provides only a summary of the work it describes, it is not clear whether D89 applied the fundamental-mode period/mean density equation to the entire RR Lyrae sample or if the equation was changed [using, say, $\left.\log P_{0}=\log P_{1}+\log (0.75)\right]$ for the $R R c$ component. Even in the latter case, however, the resulting relation turns out to be different from equation (5) in some aspects which are quantitatively small but nonetheless require investigation. Lacking the full D89 data, we shall not pursue this further here, except to emphasize the desirability of separating the RR $a b$ and $\mathrm{RR} c$ samples in future work.

Finally, it should also be mentioned that according to D89, the infrared $V-K$ RR Lyrae temperatures turn out to be cooler than those obtained from $B-V$. Unfortunately, the relevant data are again absent, so that one must await a more detailed presentation in order to tell to what extent the RRc stars would be "cooled" by use of the $V-K$ scale.

\section{PERIOD RATIOS}

The double-mode RR Lyrae pulsators (RR $d$ stars) are fairly rare. They have been found in the Draco galaxy and in the globular clusters M15, M3, IC 4499, and, recently, M68

TABLE 3

Mean Mass of $\omega$ Centauri RR Lyrae Stars FOR DIFFERENT MEAN LUMINOSITIES

\begin{tabular}{cccc}
\hline \hline$\left\langle\log L / L_{\odot}\right\rangle$ & BDE & Case 2 & D89 \\
\hline $1.71 \ldots \ldots \ldots \ldots \ldots$ & 0.49 & 0.57 & 0.62 \\
$1.76 \ldots \ldots \ldots \ldots \ldots$ & 0.59 & 0.62 & $0.73^{\mathrm{a}}$ \\
\hline
\end{tabular}

a $\left\langle\log L / L_{0}\right\rangle=1.77$. 


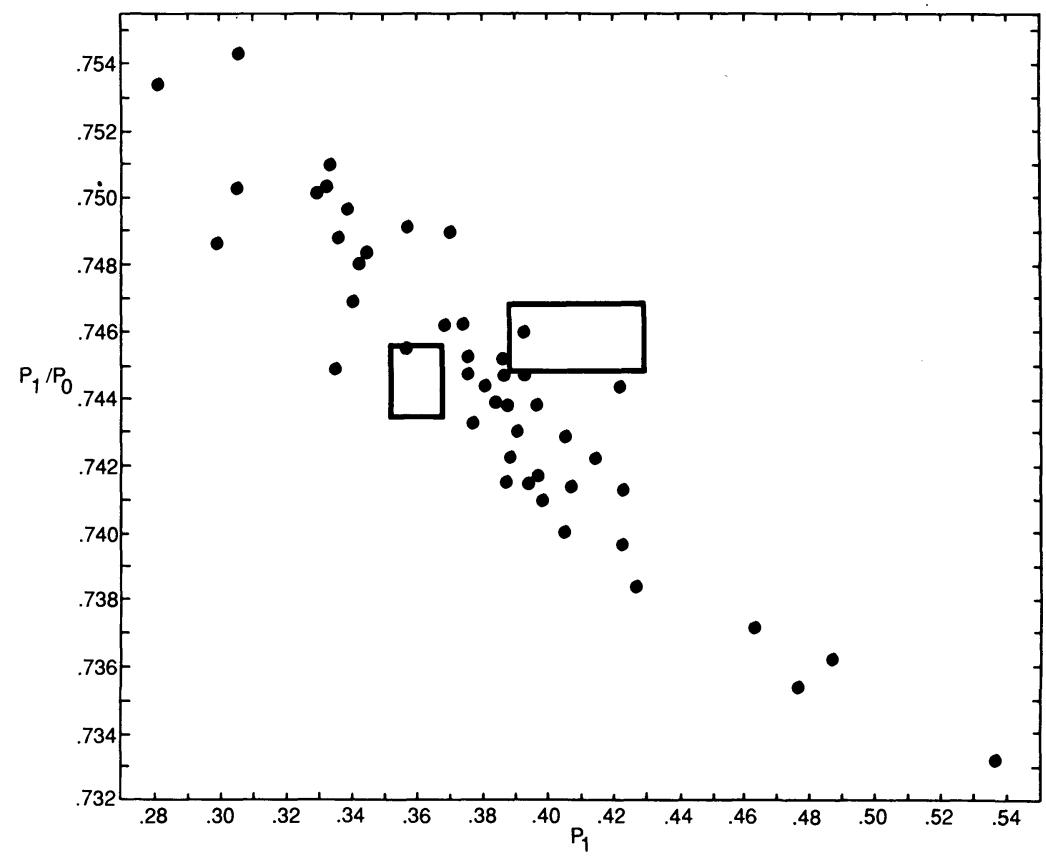

FIG. 8. $-P_{1} / P_{0}$ vs. $P_{1}$ for $47 \omega$ Cen RR $c$ stars according to case 2. Boxes indicate the RRd domains: Oo I (left box); Oo II (right box).

(Clement 1990). In the field there is only a single known example, the metal-poor star AQ Leo. Other clusters, e.g., M5 and $\omega$ Cen, seem to be devoid of these objects (Clement $e$ t al. 1986, and references therein). To discover what accounts for the presence or absence of RRd stars in a given environment would be of the utmost interest. Unfortunately, present hydrodynamic pulsation codes fail in modeling the double mode pulsators (see Kovács and Buchler 1988 for a detailed discussion). What we do know, from observations alone, is that all the RRd stars inhabit one of two domains on the Petersen diagram (period ratio vs. period). These domains correspond to the Oosterhoff I and Oosterhoff II groups, respectively.

Figure 8 renders the Petersen diagram in the form $P_{1} / P_{0}$ against $P_{1}$. The two boxes show the observed Oo I (left box) and Oo II (right box) domains according to Clement et al. (1986). The dots represent periods calculated from the parameters of our case 2 models (see Table 2). Since $\omega$ Cen does not have RRd stars, the dots ought to avoid the boxes, and to a large extent they do. Formally, only two points fall within the $\mathrm{RR} \boldsymbol{d}$ domains, and these could be explained away as due to observational error.

However, there are a number of points in Figure 8 which lie between the two boxes. Since the case 2 models occupy a more or less continuous range of mass and luminosity, it is difficult to see why the area between the boxes should be off limits for double-mode pulsation. A possible answer to this question emerges from Figure 9, which is a plot of luminosity versus mass for the models of Table 2, again given as dots. The two crosses represent the parameters for two standard linear RRd models (models A and B in Simon 1990) whose periods put them, respectively, in the Oo I $\left(M=0.55 M_{\odot}\right)$ and Oo II $\left(M=0.67 M_{\odot}\right)$ domains in the Petersen diagram. One notes that the crosses inhabit two locations which are relatively far from each other and reasonably remote from the dots. If indeed there exist two separate, remote $R R d$ areas in the luminosity-mass plane, then Figure 9 directly predicts an absence of RRd stars in $\omega$ Cen. However, this assertion cannot be definitively tested until pulsation codes become available which are adequate for modeling the double-mode phenomenon.

There is one final interesting question which emerges from Figure 9 if one accepts that the crosses mark the locations of the two domains of double mode instability and that the dots are proper representations of the $\omega$ Cen $\mathrm{RR} c$ stars. We note that near $M=0.55 M_{\odot}$, where the RRd regime would correspond to Oo I clusters like IC 4499, the dots lie above the cross. This is just what one might expect if the $\omega$ Cen stars are evolved, as lias been suggested by a number of authors (see, e.g., Gratton, Tornambé, and Ortolani 1986; D89; S89). On the other hand, near $M=0.65 M_{\odot}$ the dots lie below the cross. This implies that the higher mass $\omega$ Cen stars are less luminous than the RR Lyrae stars in an Oo II cluster such as M15. An explanation of this seeming contradiction is beyond the scope of the present investigation, but it is an important problem to be addressed in subsequent work.

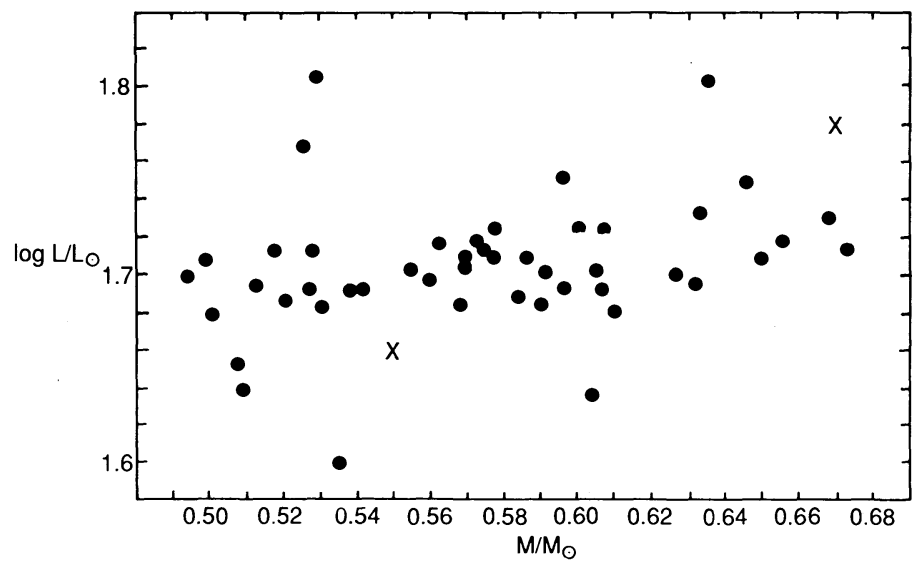

Fig. 9.-Luminosity vs. mass for $47 \omega$ Cen RR $c$ stars according to case 2 (dots). Crosses represent RRd models: Oo I $\left(M=0.55 M_{\odot}\right)$; Oo II $(M=$ $0.67 M_{\odot}$ ). 


\section{MASS AND METALLICITY}

The globular cluster RR Lyrae stars are thought to hold the key to a determination of cluster distances and ages. Particularly important in this regard is the relationship between the metallicity of an RR Lyrae star and its mass and/or luminosity. Recently, Sandage (1990) has given a number of expressions linking mass and metallicity. The first comes from the horizontal branch (HB) models of Sweigart, Renzini, and Tornambé (1987) and has the form

$$
\log M(\mathrm{RR})=-0.10[\mathrm{Fe} / \mathrm{H}]-0.318 .
$$

A second relation may be obtained if one treats the essentially bimodal distribution of RR $\boldsymbol{d}$ stars as if it were continuous. In that case (Sandage 1990), one has

$$
\log M(\mathrm{RR})=-0.10[\mathrm{Fe} / \mathrm{H}]-0.41 \text {. }
$$

While the slopes are the same in equations (6) and (7), the discrepant zero points are symptoms of a serious disagreement between pulsation and evolution theory. In this connection, Sandage (1990) raises a number of concerns regarding the RRd pulsation masses (Cox, Hodson, and Clancy 1983) which give rise to equation (7). These concerns include the possible sensitivity of the masses to changes in opacity and metallicity as well as the results of a calculation by Kovács (1985), which produced a zero point rather larger than that in equation (7).

To examine these questions, we have constructed a number of linear nonadiabatic (LNA) pulsation models in the RRd regime. We find that our models reproduce rather closely the masses of Cox, Hodson, and King (1983). These masses were also verified by Cox and Kovács separately (Cox 1988), whereas to our knowledge the original Kovács results have not been reproduced.

To test the sensitivity of the RR $d$ masses to metallicity and opacity, we have employed two models from Simon (1990). Model A $\left(M / M_{\odot}=0.55, \log L / L_{\odot}=1.66, T_{e}=7000 \mathrm{~K}\right)$ is typical of the Oo I RRd stars, while model B $\left(M / M_{\odot}=0.67\right.$, $\left.\log L / L_{\odot}=1.78, T_{e}=6950 \mathrm{~K}\right)$ represents the Oo II stars. Using these models as a base, we test the effects of alterations in metallicity and opacity. It was suggested by Simon (1982) that standard opacities may underestimate the contribution of heavy elements by a factor of $2-3$, and recent opacity calculations have given some support for this idea (Rozsnyai 1989; Iglesias, Rogers, and Wilson 1990). In the present investigation, we follow the prescription of Simon (1982) for artificially augmenting the heavy element contribution by multiplying $Z$ in the Stellingwerf (1975) formula by an arbitrary factor $f$.

Table 4 gives the results of our LNA calculations. The first row shows parameters for model A with metallicity $Z=0.001$. When the metallicity is reduced to $Z=0.0001$, the fundamental period $P_{0}$ and period ratio $P_{1} / P_{0}$ change negligibly (model A1). Next we recalculate model $A$ with an augmented metal opacity (AMO) obtained by setting $f=5$ (model A2). This is the same factor employed by Simon (1982) and shown to reduce theoretical Cepheid period ratios to observed values. Indeed, the period ratio is somewhat reduced in A2; however, as seen from model $\mathrm{A} 3$, raising the mass slightly to $0.57 M_{\odot}$ is enough to bring the parameters back into the Oo I regime.

Model A4 is calculated with a still sharper AMO, $f=10$. Here we note a substantial drop in $P_{1} / P_{0}$, which can be reversed only by a significant increase in mass and luminosity (model A5). However, an opacity augmented to the degree
TABLE 4

SOME LNA MODELS FOR THE RR $d$ STARS

\begin{tabular}{lcrcc}
\hline $\begin{array}{c}\text { Model } \\
\text { Number }\end{array}$ & $Z$ & \multicolumn{1}{c}{$f$} & $\boldsymbol{P}_{0}$ & $\boldsymbol{P}_{1} / \boldsymbol{P}_{0}$ \\
\hline $\mathrm{A} \ldots \ldots \ldots \ldots$ & 0.001 & 1.0 & 0.4838 & 0.7449 \\
$\mathrm{~A} 1 \ldots \ldots \ldots \ldots$ & 0.0001 & 1.0 & 0.4832 & 0.7452 \\
$\mathrm{~A} 2 \ldots \ldots \ldots \ldots$ & 0.001 & 5.0 & 0.4869 & 0.7433 \\
$\mathrm{~A} 3^{\mathrm{a}} \ldots \ldots \ldots \ldots$ & 0.001 & 5.0 & 0.4755 & 0.7446 \\
$\mathrm{~A} 4 \ldots \ldots \ldots \ldots$ & 0.001 & 10.0 & 0.4901 & 0.7411 \\
$\mathrm{~A} 5{ }^{\mathrm{b}} \ldots \ldots \ldots \ldots$ & 0.001 & 10.0 & 0.4791 & 0.7447 \\
$\mathrm{~B} \ldots \ldots \ldots \ldots$ & 0.0001 & 1.0 & 0.5473 & 0.7464 \\
$\mathrm{~B} 1 \ldots \ldots \ldots \ldots$ & 0.0001 & 10.0 & 0.5481 & 0.7461 \\
\hline
\end{tabular}

${ }^{\text {a }} M / M_{\odot}=0.57$

${ }^{\mathrm{b}} M / M_{\odot}=0.64, \log L / L_{\odot}=1.70$.

$f=10$ would reinstate the Cepheid period ratio problem (the ratios would now be too small!) and certainly seems unphysically large. Finally, we note that for the extremely metal poor Oo II stars, even such large increases in opacity affect the period ratios very little. This is illustrated in models B and B1.

The calculations described above demonstrate that RR $d$ pulsation masses are not significantly altered by reasonable shifts in opacity or metallicity. Uniting this result with the finding that LNA periods are sufficient for deriving the RR $d$ masses (S89; Simon 1990), we conclude that the conflict between evolution and pulsation is deep-seated and cannot be resolved by peripheral changes. Thus it would seem that if the most basic aspect of pulsation theory (i.e., the period/mean density law) is correct in its present application, the standard HB models must be wrong in some significant regard. Clearly, this statement also holds in the opposite direction.

Let us now return to $\omega$ Centauri. Figure 10 shows a plot of mass versus $[\mathrm{Fe} / \mathrm{H}]$ for 19 stars in our $\omega$ Cen sample which have metallicities published by BDE. The mass and metal abundance seem uncorrelated here, and indeed a least-squares fit to this data yields $\log M=0.008[\mathrm{Fe} / \mathrm{H}]-0.23$, a relation which certainly cannot be said to support either equation (6) or equation (7). Are the uncertainties in the masses in Figure 10 enough to accommodate, say, equation (7) despite the apparent scatter? One way to get at this is to force the masses to agree with equation (7) and see what effect this has on other results in our study. We have performed this exercise and find that the mass segregation in Figure 2 is destroyed, and the scatter significantly increased in Figure 5. Thus if the points in Figure 10 really do follow equation (7), the rather striking correlations displayed in Figures 2 and 5 must be due strictly to chance. Such a circumstance seems unlikely.

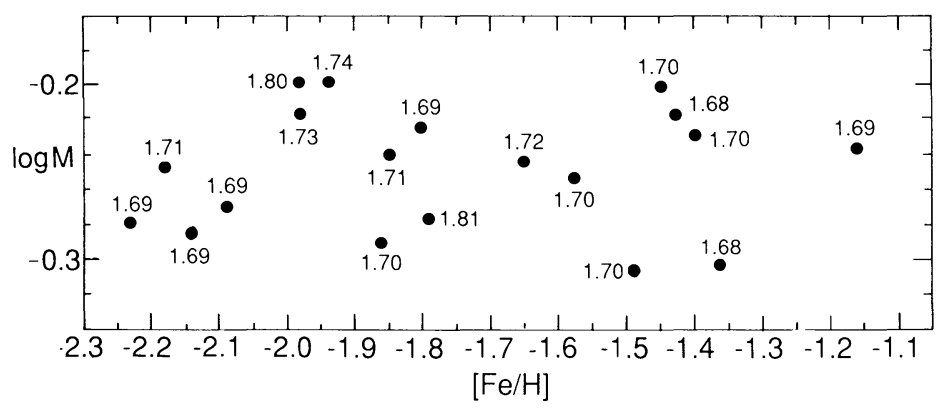

FIG. 10.-Mass (according to case 2) vs. [Fe/H] for $\omega$ Cen RR $c$ stars. The number beside each model is $\log L$, according to case 2 . 
However, there is still another point that can be made regarding Figure 10. In addition to equations (6) and (7), Sandage (1990) gives a third mass-metallicity relation

$$
\log \langle M\rangle=-0.053[\mathrm{Fe} / \mathrm{H}]-0.235,
$$

this one based on the evolutionary models of Lee, Demarque, and Zinn (1988, hereafter LDZ). The $[\mathrm{Fe} / \mathrm{H}]$ dependence is weaker here due to late redward evolution which carries lower mass stars into the instability strip to join higher mass stars of the same metallicity. While a number of authors have raised objections to the LDZ models as a scenario for understanding the Oosterhoff dichotomy (e.g., Rood and Crocker 1989; Buonanno, Corsi, and Fusi-Pecci 1989; Sandage 1990), our purpose here is to consider whether late evolution as in LDZ can explain the smearing out of the masses versus metallicity in $\omega$ Centauri.

To be more specific, one may note from standard zero-age horizontal branch models (e.g., LDZ; Sweigart 1987) that within the narrow temperature confines of the RRc instability strip, only a very small mass range, $\Delta M \sim 0.03 M_{\odot}$, is allowed at given metallicity. On the other hand, the spread of masses we find among the $\omega$ Cen $R R c$ stars is nearly 5 times as large. It seems that this contradiction can be explained within the framework of the standard models only if the stars of lower mass are in very late stages of $\mathrm{HB}$ evolution. If this explanation is correct, then it may be deduced from the LDC evolutionary tracks that, at given $[\mathrm{Fe} / \mathrm{H}]$, the stars along the bottom of Figure 10 (lower mass) ought to be brighter than the stars along the top (higher mass) by a large amount, say $\Delta \log L \sim 0.06$. That this is not the case is clearly indicated by the numbers in the body of Figure 10, which give the value of $\log L$ for each star according to case 2 . We conclude that if the analysis in the present investigation is correct, there is no relationship between mass and metallicity among the $R R c$ stars in $\omega$ Cen.

Perhaps the lack of a mass-metallicity correlation should not be surprising, since it also found for the RR Lyrae field stars in the Baade-Wesselink (BW) analyses of Cacciari, Clementini, and Busser (1988) and of Liu and Janes (1990). The latter authors mention this result explicitly but do not discuss it. However, a partial comparison may be made between our method and the Baade-Wesselink treatment for three RRc stars, namely DH Peg (Jones, Carney, and Latham 1988), YZ Cap (Cacciari, Clementi, and Busser 1988) and T Sex (Liu and Janes 1990). The second and third columns of Table 5 give masses and luminosities of these stars according to the $\mathrm{BW}$ analysis. In column (4) we display values of $\phi_{31}$ obtained from Simon and Teays (1982) or Simon (1988b). Let us adopt the BW mass for each star and use it along with $\phi_{31}$ and $Y=0.25$ (the result will be very little changed if we choose a different $Y$ ) in equation (1) to obtain $\log L\left(\phi_{31}\right)$. These values are listed in column (5) of Table 5 . We note the agreement of $\log L(\mathrm{BW})$ and $\log L\left(\phi_{31}\right)$ at approximately the level expected from the uncertainty in equation (1).

Though we have been able to make this comparison in only the three cases indicated, we shall now argue that the agreement we find is significant. Using the period/mean density relation (eq. [5]) along with equations (8) and (13) from Liu and Janes (1990) and the definitions of absolute magnitude and effective temperature, it is easy to show that

$$
\log L=0.75 \log M+K_{1},
$$

is the relationship that must hold when the BW technique is
TABLE 5

BAADE-WESSELINK VERSUS $\phi_{31}$ LUMINOSITIES FOR THREE RR $c$ STARS

\begin{tabular}{ccccc}
\hline $\begin{array}{c}\text { Star } \\
(1)\end{array}$ & $\begin{array}{c}M(\mathrm{BW}) \\
(2)\end{array}$ & $\begin{array}{c}\log L(\mathrm{BW}) \\
(3)\end{array}$ & $\begin{array}{c}\phi_{31} \\
(4)\end{array}$ & $\begin{array}{c}\log L\left(\phi_{31}\right) \\
(5)\end{array}$ \\
\hline DH Peg $\ldots \ldots \ldots$ & 0.55 & 1.54 & 2.85 & 1.57 \\
YZ Cap $\ldots \ldots \ldots$ & 0.53 & 1.58 & 2.71 & 1.53 \\
T Sex $\ldots \ldots \ldots \ldots$. & 0.47 & 1.59 & 3.94 & 1.56 \\
\hline
\end{tabular}

applied to a given star. That is, if $\log M(\mathrm{BW})$ is the mass determined by the $\mathrm{BW}$ analysis, then $\log L(\mathrm{BW})$ will be given by equation (9). We note that $K_{1}$ is a constant involving the period and " photometric angular diameter" and is thus different for different stars.

The corresponding relation for the $\phi_{31}$ analysis comes directly from equation (1) and has the form

$$
\log L=1.8 \log M+K_{2},
$$

where $K_{2}$ involves $\phi_{31}$ and thus also varies from star to star. Given that equations (9) and (10) have such different slopes, it is difficult to see how both could be satisfied (as in Table 5) for each of three stars unless the listed masses and luminosities are the true stellar parameters, arrived at in different ways by the two different analyses. Unfortunately, since the $\phi_{31}$ method is not applicable to RRab stars, further comparisons must await an enlarged sample of the $\mathrm{RR} c$ stars subjected to $\mathrm{BW}$ analysis. In the meantime, the two techniques seem to agree on the result that there is no mass-metallicity relation among the $R R$ Lyrae stars.

\section{LUMINOSITY, METALLICITY, AND CLUSTER AGES}

In a recent article, Sandage and Cacciari (1990, hereafter SC) give a detailed discussion of globular cluster ages. The age of a cluster can be found once the main-sequence turnoff luminosity, $M_{\mathrm{BOL}}(\mathrm{TO})$, is known. The determination of $M_{\mathrm{BOL}}(\mathrm{TO})$ proceeds either directly by main sequence fitting or via the method preferred by SC and many other authors, namely the use of RR Lyrae stars to set down a true cluster luminosity scale.

The employment of this latter method has evolved into the form of a luminosity-metallicity relation for the RR Lyrae stars,

$$
M_{V}(\mathrm{RR})=a[\mathrm{Fe} / \mathrm{H}]+b,
$$

with the spread of globular cluster ages depending critically on the slope $a$. Indeed, SC have shown that $a=0$ implies an age spread $\Delta \tau$ as large as $10 \mathrm{Gyr}$, while $a \sim 0.2$, implies $\Delta \tau \sim 4$ Gyr, and $a \sim 0.4$ is consistent with virtually zero spread in globular cluster ages.

The reality of the dependence of $M_{V}(\mathrm{RR})$ on $[\mathrm{Fe} / \mathrm{H}]$ (i.e., $a>0$ ) is argued strongly by SC and by Sandage (1990). However, the modeling of the RRd stars, on the one hand, and a BW analysis of RR Lyrae field stars, on the other, imply quite different values of $a$-namely, about 0.4 in the former case and 0.2 in the latter. Furthermore, in the one instance in which equation (11) may be directly tested, namely in $\omega$ Centauri, no dependence of luminosity on metallicity may be discerned. We emphasize again that the null result in $\omega$ Cen is not due to late evolution as in LDZ, since in that case the lower mass stars should be brighter, a condition which is certainly not seen in Figure 10. 
In addition, as we have shown, both the mass zero point and mass-metallicity relation implied by current HB models are in conflict with results from pulsation theory and from BW analysis. To the extent that the HB models are called into question, the theoretical underpinning of a brightnessmetallicity relation for $\mathrm{HB}$ stars is removed. Perhaps, then, there does not exist a universal relation between $M_{V}$ and $[\mathrm{Fe} / \mathrm{H}]$. Or perhaps such a relation is mediated by the longsought "second parameter." The horizontal branch may well be more complex that we have assumed.

In the face of such uncertainties, it is probably not advisable to use a general brightness-metallicity relation to derive cluster ages. However, if one takes the theory of the RRd stars to be the most reliable guide to RR Lyrae luminosities, a variation of the SC method can be employed which does not invoke equation (11). We shall illustrate this briefly in the case of M15. According to Table 8 of Bingham et al. (1984), the average $V$ magnitude of $10 \mathrm{RR} d$ stars listed by Nemec (1985) is $V(\mathbf{R R})=15.84$. If we deredden this magnitude, we find with the aid of Table 3 of SC a difference between the mean RR Lyrae and main-sequence turnoff magnitudes, $\Delta V=3.46$. We now assign our mean RR $d$ star a "fiducial" Oo II RR $d$ luminosity, $\log L(\mathrm{RR} d)=1.77$ (Cox, Hodson, and Clancy 1983; Nemec 1985), or $M_{\text {BOL }}(\operatorname{RR} d)=0.325$. Finally, with HB and TO bolometric corrections as given by $\mathrm{SC}$ and using the $\mathrm{SC}$ equation (13) with $Y=0.24$, we find, for the age of M15, $14.8 \mathrm{Gyr}$. Had we adopted $\log L(\operatorname{RR} d)=1.78$, the M15 age would have been $14.5 \mathrm{Gyr}$, while $\log L(\mathrm{RR} d)=1.76$ would have yielded 15.2 Gyr.

As expected, the age we determine for M15 agrees with the age given in the last column of Table 3 of SC, since the crucial component of the latter is the RR $d$ masses. Thus if one believes elementary pulsation theory, the age of M15 (without oxygen enhancement) is about $15 \mathrm{Gyr}$, irrespective of any assumption regarding the $H B$. The same statement may be made for the RRd age of M3 which SC find to be 15.5 Gyr. Recently, Clement (1990) has suggested that as many as 10 RRd stars exist in the Oo II cluster M68, and has determined the mass of one of them to be $M(\mathrm{~V} 3)=0.64 M_{\odot}$, fully in line with the RRd masses in M15. The RRd age given by SC for M68 is $14.5 \mathrm{Gyr}$. Thus the ages determined for three of the four clusters known to contain RR $d$ stars (the other cluster is IC 4499) seem to span a range no greater than 1 Gyr. Once again, we emphasize that this result is independent of any assumptions concerning $\mathrm{HB}$ evolution.

What about clusters which do not have RR $d$ stars? In such cases, one may use the $\phi_{31}$ diagnostic to calculate ages, as is illustrated for $\omega$ Cen in what follows. The derivation is similar to that for M15. We consider the 29 star $R R c$ sample in common between BDE and the present study. We calculate from BDE the average $V$ magnitude for this sample, obtaining $V(R R)=14.53$. The average luminosity for the same stars is $\log L(\mathrm{RR})=1.70$, according to case 2 . Dereddening $V(\mathbf{R R})$ and using data from Table 3 of $\mathrm{SC}$, we find $\Delta V=3.68$, in precise agreement with the $\mathrm{SC}$ value. Combining our log $L(R R)$ with data from Table 3 of SC and using the SC equation (13), we obtain finally an age for $\omega$ Cen of $17.3 \mathrm{Gyr}$ as opposed to the SC value (last column of their Table 3) of 19.2 Gyr. The difference in age comes about because our RR Lyrae luminosity is brighter than that used by SC. Or, to put it another way, if our analysis is correct, $\omega$ Cen does not fit the $M_{V}$ versus $[\mathrm{Fe} / \mathrm{H}]$ relation obtained by Sandage $(1990)$ from the RRd stars.

\section{FINAL REMARKS}

We have seen that the present work along with $\mathbf{S} 89$ derives RR Lyrae masses which agree with those of the RR $d$ stars. However, it is also true that in a number of important areas, our results are contrary to those of other investigations. Among these disagreements are the following: (1) Our RR Lyrae masses are considerably smaller than those derived for $\omega$ Cen by D89. The latter masses are much more in line with current HB models which, it seems, cannot be correct if our results are correct; (2) The present study suggests that the standard $B-V$ temperature scale (e.g., VandenBerg and Bell 1985) has much too sharp a slope, at least when applied to the $\mathrm{RR} c$ pulsators. This implies that static model atmospheres may not be applicable in the RRc case; and (3) We find no massmetallicity relation among the $\omega$ Cen $R R c$ stars. This result seems to call into further question any facile interpretation of the metallicity as the key parameter governing the horizontal branch.

In view of the important points involved here, it is worthwhile to briefly reexamine the basis for the results of the present study. The pivotal ingredients are equations (1) and (5), derived from our theoretical models, along with our choice of the case 2 mass scale. Equation (5) is merely the period/mean density relation, the most fundamental in pulsation theory. If this is incorrect, then we understand very little about stellar pulsations. The other relation, equation (1), is also very likely to be (approximately) right. Use of this expression reproduces the observed rise of $\phi_{31}$ with period, including the value of the slope $\left(\partial \phi_{31} / \partial \log P\right)_{M} \cong 6$. It also yields in combination with the case 2 scale: (1) a mass range which agrees with that of the RR $d$ stars and the BW masses of field RR Lyrae stars (e.g., Liu and Janes 1990); (2) temperatures which are consistent with the location of the $\omega$ Cen RRc stars in the overtone instability strip; and (3) an explanation for the absence of RR $d$ stars in the $\omega$ Cen cluster. If equation (1) is wrong, these results must be considered strictly fortuitous.

On the other hand, equation (4) was invented to "fix up" the coefficient of $\log M$ in equation (2). The range of $Y$ implied by the former equation is not large (see Table 1), and a relationship such as that in equation (4) could result, for example, if mass loss preceded or coincided with dredge-up (Faulkner and Iben 1967; Sweigart 1987) during the initial red giant phase. In that case, the envelope with lower mass would be proportionately more enriched by the same amount of dredged-up helium. Perhaps other reasonable scenarios could also be invented to accomplish the same thing.

Be this how it may, one still must view equation (4) and the mass and helium coefficients in equation (2) as somewhat uncertain. This means that the helium abundances given in Table 2 ought to be taken as tentative at the present time. We reiterate, however, that the other results of this investigation depend upon equations (1) and (5) and thus should be considered much more secure. In any event, as indicated in S89, the consistency of our theory can be tested further by its application to the $\mathrm{RR} c$ stars in other clusters. This is the next task that must be undertaken.

The author wishes to thank the University of Nebraska for a Maude Hammond Fling Fellowship, without which this work would not have been completed. I am also grateful to the National Science Foundation for providing computer time on 
the Cray X-MP at the National Center for Supercomputing Applications, to J. O. Petersen for furnishing a table of $\omega$ Cen data, and to J. M. Nemec for interesting discussions. Finally, the present work was greatly aided and inspired by the Invited Talks of R. J. Dickens, R. T. Rood and D. A. Crocker, and A. Sandage delivered at $I A U$ Colloquium 111.

\section{REFERENCES}

Bingham, E. A., Cacciari, C., Dickens, R. J., and Fusi-Pecci, F. 1984, M.N.R.A.S., 209, 765 .

Buonanno, R., Corsi, C. E., and Fusi-Pecci, F. 1989, Astr. Ap., 216, 80.

Butler, D., Dickens, R. J., and Epps, E. A. 1978, Ap. J., 225, 148 (BDE)

Cacciari, C., Clementini, G., and Busser, R. 1988, Astr. Ap., 209, 154.

Clement, C. M. 1990, A.J., 99, 240 .

Clement, C. M., Nemec, J. M., Robert, N., Wells, T., Dickens, R. J., and Bingham, E. A. 1986, A.J., 92,825

Cox, A. N. 1988, in IAU Colloquium 95, Second Conference on Faint Blue Stars, ed. A. G. Davis Phillip, D. S. Hayes, and J. W. Liebert (Schenectady: L. Davis Press), p. 161.

Cox, A. N., Hudson, S. W., and Clancy, S. P. 1983, Ap. J., 266, 94.

Dickens, R. J. 1989, in The Use of Pulsating Stars in Fundamental Problems of Astronomy, ed. E. G. Schmidt (Cambridge: Cambridge University Press), p. 141 (D89).

Faulkner, J., and Iben, I. 1967, Nature, 215, 44.

Gratton, R. G., Tornambé, A., and Ortolani, S. 1986, Astr. Ap., 169, 111.

Iglesias, C. A., Rogers, F. J., and Wilson, B. G. 1990, preprint.

Jones, R. V., Carney, B. W., and Latham, D. W. 1988, Ap. J., 332, 206.

Kovács, G. 1985, Acta Astr., 35, 37.

Kovács, G., and Buchler, J. R. 1988, Ap. J., 324, 1026.

Lee, Y.-W., Demarque, P., and Zinn, R. 1988, in IAU Colloquium 95, Second Conference on Faint Blue Stars, ed. A. G. Davis Phillip, D. S. Hayes, and J. W. Liebert (Schenectady: L. Davis Press), p. 137 (LDZ).

Liu, T., and Janes, K. A. 1990, preprint.

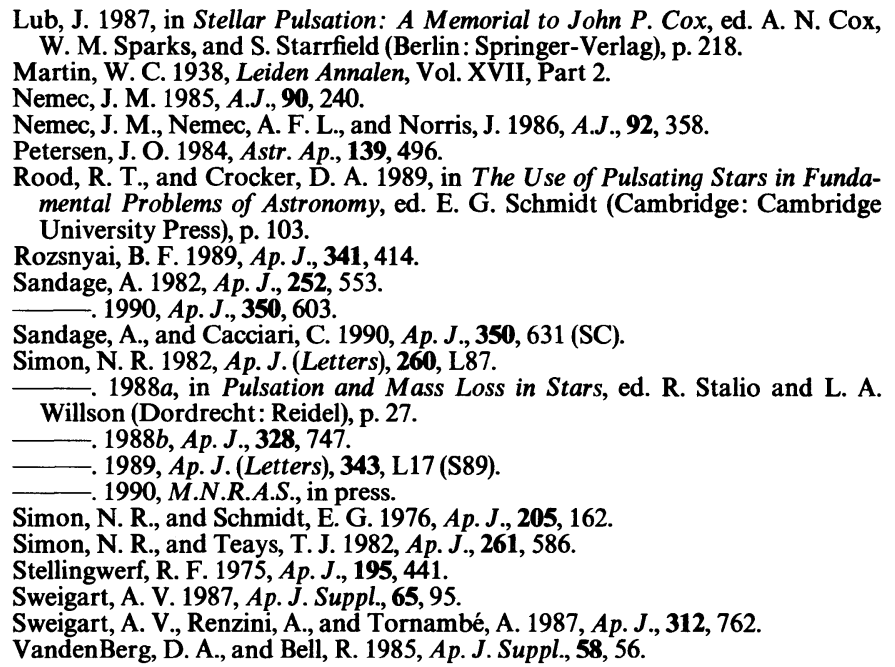

NoRman R. Simon: Department of Physics and Astronomy, University of Nebraska, Lincoln, NE 68588-0111 